\title{
Low-Cost TDRSS Communications for NASA's Long Duration Balloon Project
}

\author{
David J. Israel \\ Code 531.2/RF Systems Section \\ NASA/Goddard Space Flight Center \\ Greenbelt, MD 20771
}

\begin{abstract}
A new transponder and RF ground support equipment are making Tracking and Data Relay Satellite System (TDRSS) communications available to lower budget NASA projects. Due to their use of current and, in some cases, state-of-the-art technology, the new designs provide advanced features to the user and are still less expensive than any previous designs. The new equipment will be used first to support long duration scientific balloon flights in Antarctica in December 1992.
\end{abstract}

Introduction

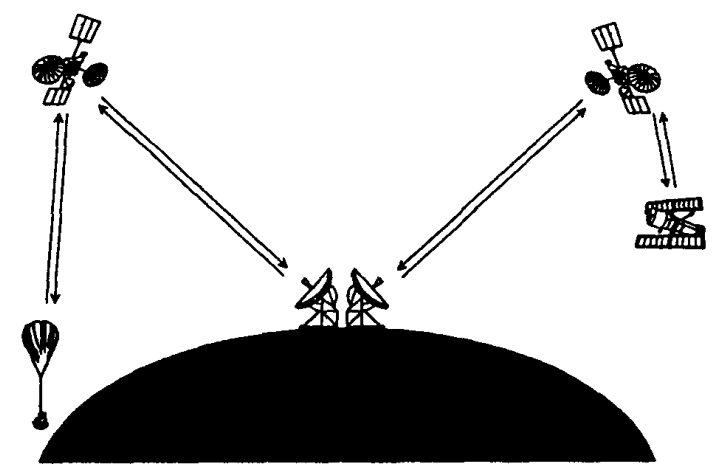

Fig. 1. The NASA Tracking and Data Relay Satellite System

\section{INTRODUCTION}

NASA's TDRSS allows unparalleled flexibility for spacecraft users to conduct spacecraft mission operations by providing the potential for two-way communications for more than $85 \%$ of their orbits. The baseline components of TDRSS

Based on a presentation at NTC ' 92

U.S. Government work not protected by U.S. Copyright (see Figure 1) are two Tracking and Data Relay Satellites, TDRS-East and TDRS-West, in geosynchronous orbit and a ground terminal in White Sands, New Mexico. Commands are sent via the forward link that originates at the ground terminal and are relayed by a TDRS to the user spacecraft. The user spacecraft transmits its scientific and engineering data to a TDRS that relays this return link to the ground terminal. Each TDRS supports S-band Multiple Access with a phased array antenna and S- and Ku-band Single Access with either of two 4.8-meter parabolic antennas. Return data rates of up to $3 \mathrm{Mbps}$ can be supported through an S-band link and up to $300 \mathrm{Mbps.,}$ can be supported through a Ku-band link. TDRSS can also provide tracking services within an accuracy of 150 meters.

Complexity and expense have, until now, limited TDRSS users to primarily the Space Shuttle, the Great Observatories, and several other mainline NASA spacecraft projects. A new low-cost non-space-qualified TDRSS user S-band transponder and set of RF ground support equipment have brought TDRSS capabilities to a class of users who seek enhanced operations for low budget scientific investigations. The Goddard Space Flight Center (GSFC)/Wallops Flight Facility's Long Duration Balloon Project (LDBP) will be the first to take advantage of the new TDRSS user hardware for communications through the TDRSS S-Band multiple access system during their around-the-world scientific balloon flight campaigns. The Balloon-Class TDRSS User Transponder has been developed by Stanford Telecom for GSFC in a program sponsored by NASA Headquarters' Office of Space Communications' Advanced Systems Program (Code OP). The TDRSS User RF Test Set (TURFTS) has been developed by GSFC's RF Systems and Embedded Systems Sections. Both designs achieve better performance and increased capabilities than their predecessors at a reduced cost. The Balloon Transponder is one-tenth the cost of the current generation space-qualified transponder and TURFTS is less than one-half the cost of the currently available transponder test equipment. 


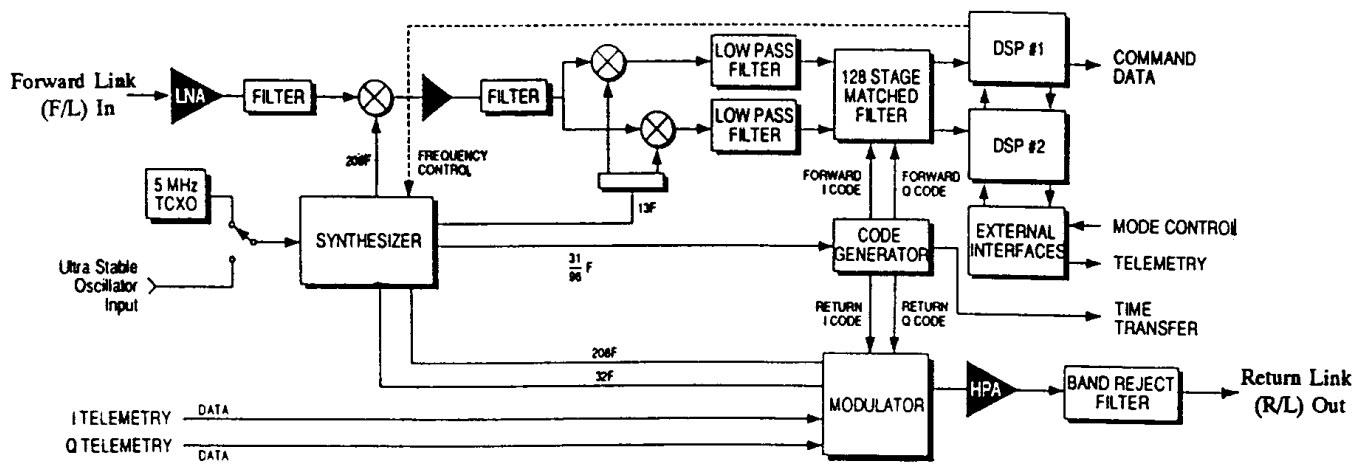

Fig. 2. Balloon-Class TDRSS User Transponder Block Diagram

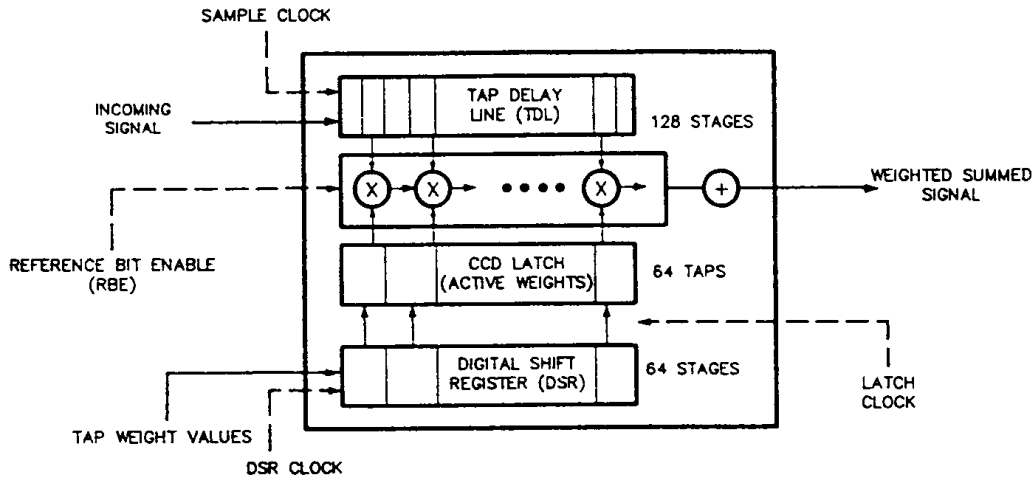

Fig. 3. Single Channel of the 4 ABC CCD Correlator Chip

\section{BALLOON-CLASS TDRSS USER TRANSPONDER}

TDRSS employs direct sequence spread spectrum signalling to provide code multiplexing, ranging, interference rejection, and reduced spectral flux density. The user transponder must therefore be able to acquire and demodulate a spread forward link and transmit a spread return link. The new Balloon Class Transponder uses programmable charge coupled device (CCD) correlators and digital signal processing (DSP) chips in a highly integrated spread spectrum receiver design. A block diagram of the Balloon Transponder is seen in Figure 2. The design has many advanced features including fast signal acquisition, high data rate capabilities, a statistical forward link data quality monitor, and reduced power consumption.

The programmable CCD correlator chip incorporated into the transponder design is the Lincoln Labs $4 \mathrm{ABC}$. One of the four CCD channels of the $4 \mathrm{ABC}$ is diagrammed in Figure 3. The incoming signal is sampled and shifted at a rate up to 40 Msps through the analog 128-stage tap delay line. Tap weight values are preloaded into the Digital Shift Register and clocked into the CCD Latch to become the active tap weights. The Reference Bit Enable together with the tap weight values controls the length of the correlator. Every other sample is weighted by $a \pm 1$ or 0 and summed at each clock period to generate the weighted summed signal. The analog correlations provide a dynamic range of $66 \mathrm{~dB}$ and nonlinearity below -50 $\mathrm{dB}$. This is the equivalent of a digital correlator with $\sim 11$-bit resolution without quantization. The $4 \mathrm{ABC}$ is fabricated using $4 \mu \mathrm{m}$ NMOS technology and is packaged in a 68-lead pin grid array. Its total power dissipation is only $1 \mathrm{~W}$.

The TDRSS forward link is a staggered quaternary phase shift keyed (SQPSK) signal. The in-phase component is known as the command channel and the quadrature-phase component is known as the range channel. Each channel has its own PN code. The command channel PN code, the short code, is $2^{10-1}$ chips long and the range channel PN code, the long code, is $256 \times\left(2^{10}-1\right)$ chips long. The Balloon Transponder uses three out of the four $\mathrm{CCD}$ channels of a single, $4 \mathrm{ABC}$ chip. Channels CCD-A and CCD-B perform correlations on the in-phase and quadrature-phase components of the command channel, while channel CCD-D performs correlations on the in-phase component of the range channel. The analog CCD outputs are converted to 8-bit digital values that are processed by two 24bit fixed-point digital signal processors. The two DSP chips perform all of the acquisition and tracking algorithms including the PN code, carrier, and bit sync loops and provide the control and telemetry interfaces.

For short code acquisition, 64 adjacent chip phases are loaded simultaneously as the tap weights for CCD-A and CCD-B. The input signals are sampled at twice the chip rate $(\sim 6 \mathrm{MHz})$, so 
128 half-chip code phases are tested in parallel. The tap weights are updated every 64 chips in order to keep the same code phase in each stage. After a fixed dwell period, the local code is delayed by 64 chips and the next 64 chip phases are loaded into the CCDs as the new tap weight. After sixteen 64-chip windows have been tested, the whole 1023-chip code has been searched. The processor then selects the local PN code phase to be the phase that produced the largest correlation value. This technique produces short code acquisition times of 2.2 seconds for a $125 \mathrm{bps}$ data rate down to 50 milliseconds for a $24 \mathrm{Kbps}$ data rate under threshold conditions $(\mathrm{C} / \mathrm{No} \geqslant 33 \mathrm{~dB}-\mathrm{Hz}$; frequency offset $\leqslant 1500 \mathrm{~Hz}$; doppler rate $\leqslant 70 \mathrm{~Hz} / \mathrm{sec}$ ) with $90 \%$ acquisition probability. This is an improvement over the current generation TDRSS user transponder that requires up to 20 seconds for short code acquisition at threshold and can only receive data rates up to $1000 \mathrm{bps}$.

After successful short code acquisition, carrier acquisition is begun using the outputs from CCD-A and CCD-B, Carrier acquisition is achieved in $128 \mathrm{~ms}$ or less using complex Fast Fourier Transforms (FFT) to locate the carrier to within the carrier tracking loop's pull-in range. The maximum frequency offset is $1500 \mathrm{~Hz}$, while the carrier tracking loop bandwidth is $60 \mathrm{~Hz}$ or less depending on the data rate. The CCD outputs are accumulated in two predetection accumulators and sampled at a rate large enough to provide the necessary resolution. Unlike previous transponders, carrier acquisition is possible even when data modulation is present. For high data rates ( $\geqslant 1 \mathrm{Kbps}$ ), the data modulation is removed by complex squaring of the samples. These values are used as the quadrature samples for the complex FFT. The frequency that corresponds to the peak magnitude of the FFT is selected as double the frequency offset value. To avoid squaring losses for low data rates when the $\mathrm{C} /$ No is low, the accumulator outputs are not squared and the resulting complex FFT spectrum is the input signal spectrum which includes the data modulation. This spectrum could be anything from a spike if no modulation is present to a sinc [2] function if data is present. An FIR filter with tap values having the shape of a sinc [2] function with the first null at the data rate is used to provide a maximum likelihood estimate of the frequency offset. Since the statistical power spectrum of a BPSK random process is a sinc [2] function, the output from the FIR filter, after the FFT values have been passed through it, will have a peak at the offset frequency value.

Range code acquisition is accomplished by a sequential threshold search using the CCD-D correlator. Since there is no data on the range channel and the carrier has been acquired, only the in-phase component of the range channel is necessary for correlation to the CCD. Parallel correlation tests are not feasible because the possible code phases are separated by 1023 chips, which is greater than the CCD window. However, the range code is synchronized to the command code, which has already been acquired, so there are only 256 possible code phases to be tested. A possible code phase is tested until its correlation measurement falls below the lower threshold, leading to dismissal, or crosses the upper threshold, leading to acceptance. This method acquires the usage code with $90 \%$ probability in less than 10 seconds at a minimum $\mathrm{C} /$ No of $23 \mathrm{~dB}-\mathrm{Hz}$.

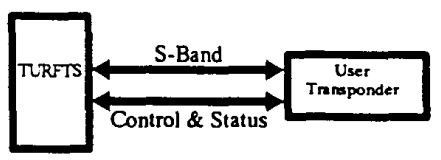

1) Benchtop Transponder Testing

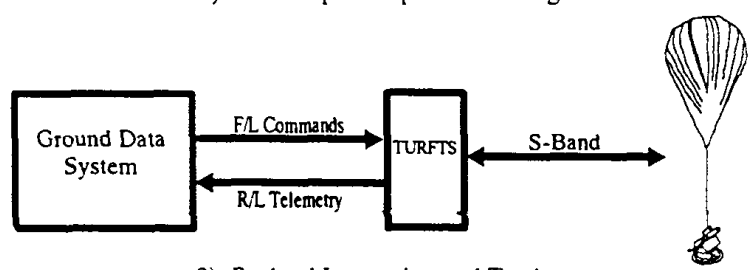

2) Payload Integration and Testing

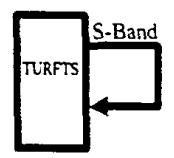

3) TURFTS Self-Testing

Fig. 4. TURFTS Operating Configurations

PN code, carrier, and symbol tracking are all achieved by normalized digital tracking loops. The normalization eliminates the need for coherent automatic gain control. During tracking operations, the CCD tap weights are updated every 64 chip periods to provide a $1 / 2$-chip early, on-time, and $1 / 2$-chip late correlation value as the first three outputs every 64 chips. This de-spreads the input signal and provides the necessary data to the PN code tracking loop, which is a digital delay lock loop. The CCD sample clock is adjusted to close the PN code tracking loop. $A \tan (\theta)$ discriminator provides the phase error estimate for carrier tracking. The $\tan (\theta)$ function is self-normalizing and also achieves better pull-in performance than a $\sin (\theta)$ discriminator. The output frequency of a numerically controlled oscillator is updated to close the carrier tracking loop. Symbol tracking is accomplished by a data transition tracking loop which adjusts the timing of the CCD latch update signal such that it coincides with a data transition.

Another advanced feature of the Balloon Transponder is that it reports an estimate of its input bit error rate. A DSP produces this estimate by first computing the ratio of the input received narrowband power over the received wideband power and then using that result to index a look-up table. The estimate is provided in a three-bit field which indicates the magnitude of the bit error rate from $10^{-6}$ to $10^{-2}$.

The transponder includes a $5 \mathrm{~W}$ transmitter designed to meet the TDRSS user signal constraints. Size, weight, and power savings are accomplished by sharing common synthesizer circuits between the receiver and transmitter. The Balloon Transponder weighs only 12.5 pounds, its total power 

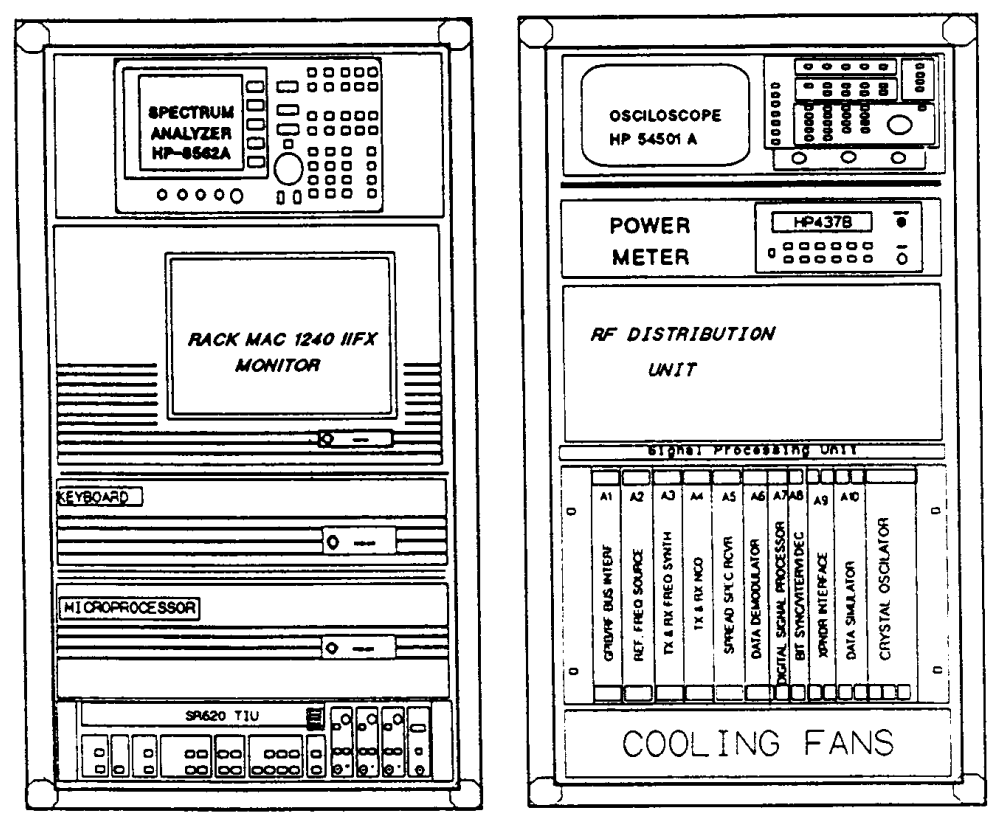

Fig. 5. TDRSS User RF Test Set (TURFTS)

consumption is $37.2 \mathrm{~W}$, and its size is $13.5 \times 7.44 \times 3.94$ in [3].

Though it is not space-qualified, the Balloon Transponder is designed to meet the LDBP flight requirements. These requirements include withstanding $10 \mathrm{G}$ 's $(50 \mathrm{~Hz}$ to $4000 \mathrm{~Hz})$ sinusoidal vibration, $10 \mathrm{G}$ 's of static acceleration, $25 \mathrm{G}$ 's of pyrotechnic shock, atmosphere at altitudes up to 150,000 feet, and temperatures from -40 to $65^{\circ} \mathrm{C}$. The transponder is also designed to minimize radiation susceptibility and to detect and recover from a single event upset.

\section{TDRSS USER RF TEST SET (TURFTS)}

A TDRSS user requires a means to characterize its transponder's performance in a laboratory environment and to link its ground and flight data and command systems for preflight testing at the integration facility or launch site. TURFTS is a set of RF ground support equipment that can provide both the RF communications and direct control and monitoring necessary for transponder testing and a two-way RF link for preflight testing. The LDBP launches its balloons from various remote sites including Antarctica and will be reflying its transponders. For these reasons, transportability was an important consideration for the TURFTS design. The TURFTS design takes advantage of the current technology to create a test set that is more functional and transportable than previous test sets.

The three basic operating configurations for TURFTS are seen in Figure 4. In the first configuration, the user transponder is directly connected to TURFTS. TURFTS transmits an $\mathrm{S}$-Band forward link to the transponder and receives the S-Band return link. TURFTS also directly controls the transponder and monitors its status indicators through connections to the control, command, and telemetry interfaces. Through these connections, transponder control and response indicators can be incorporated into automated test sequences, which, along with computer control of the TURFTS' forward link power level, frequency, and frequency sweep rate, eases the characterization of the transponder receiver's acquisition and tracking performance. TURFTS can also simulate forward link command data or return link telemetry data and provide a bit error rate measurement. A time interval counter, which is included with TURFTS, can measure the transponder ranging delays and an oscilloscope and spectrum analyzer are also included to test the transponder transmitter's performance and monitor various signals within TURFTS.

In the second configuration, TURFTS is used to link the project flight system to the ground data system using either an antenna or cable RF link. The commands from the ground data system are transmitted on the TURFTS S-Band forward link to the flight system. The return link is received by TURFTS, which provides the demodulated and decoded data and the recovered data clock to the ground data system. This configuration permits some payload integration and preflight testing to be accomplished without the need for actual TDRSS service.

In the third configuration, a frequency translator is used to loop the TURFTS transmitter back to the TURFTS receiver. 
Any of the forward or return link TDRSS User PN codes may be used. This configuration allows for verification of the TURFTS RF links and components in order to assist in the isolation of problems during troubleshooting.

TURFTS is packaged in two 4-1/2 foot tall racks (Figure 5). This is less than half the size of past test set designs. The racks are shock-mounted packing cases allowing TURFTS to be easily transported and configured. The first rack contains the System Controller, a time interval counter, and a spectrum analyzer. The other rack contains an oscilloscope, an RF power meter, the RF Distribution Unit (RFDU), and the TURFTS Signal Processing Unit (TSPU). The RFDU and TSPU are custom designed units while the rest of the test set components are commercially available equipment.

The TURFTS System Controller is a Macintosh IIfx computer executing National Instruments' LabVIEW software. LabVIEW provides a graphical user interface for the operator to control the test set and display the status and test results. The operator is able to automate transponder test sequences and design custom screen displays. The Macintosh communicates to the rest of TURFTS through a GPIB bus, which is standard on the commercially available equipment and designed into the custom equipment.

The RFDU is a custom designed S-Band RF chassis that provides the RF interface between the transponder and TURFTS. The RFDU contains the QPSK modulator and the frequency multipliers, down-converters, and translator. Various RF switches and attenuators in the RFDU can be controlled by the System Controller to automatically configure the signal paths and power levels.

The TSPU is a VME chassis housing custom analog and digital modules. Seven modules, A2 through A8, comprise the TURFTS Receiver and Transmitter by performing all of the frequency synthesis, IF, and baseband functions. Modules A2 through A4 accomplish the frequency synthesis and PN code generation. Combined direct digital synthesis and phase-locked loops techniques are used to produce the receiver and transmitter local oscillators. Numerically Controlled Oscillator (NCO) VLSI chips provide frequencies up to $25 \mathrm{MHz}$ that are coherently translated up to the intermediate frequencies by phase-locked loops. The PN codes are provided by custom VLSI TDRSS PN Code Generator chips. Each chip can generate all 85 TDRSS user forward and return link codes, including those necessary for PN code tracking, and is processor controllable. The Tracking IF Module (A5) de-spreads the receiver IF and provides the de-spread IF to the Data Demodulator Module (A6) and two quadrature baseband pairs to the DSP Module (A7). The Data Demodulator Module passes the demodulated data to the Bit-Sync and Decoder Module (A8) where bit synchronization and Viterbi decoding are performed using commercially available VLSI chips. At this point, the I and $Q$ channel demodulated and decoded data streams and the recovered data clocks are output.

The DSP Module contains a 32-bit floating-point DSP chip that executes the PN code acquisition and tracking, carrier acquisition and tracking, and automatic gain control (AGC). The DSP also controls the setting and sweeping of the transmitter and receiver frequency synthesizers. The TURFTS receiver is designed to operate under test conditions where the signal-to-noise level is high enough to allow simpler DSP algorithms to be practical. This allows the single DSP chip to execute all of the required functions in real time. PN code acquisition is accomplished by a fixed dwell time threshold test. A digital tau-dither loop tracks the PN code, and digital loops are used for both carrier acquisition and tracking and AGC. When a transmitter frequency sweep is occurring, the DSP sweeps the receiver center frequency along with the transmitter center frequency. This prevents the receiver carrier loop from having to track the sweep when the transponder coherently sweeps its return link.

The other three modules in the TSPU are the GPIB Interface Module (A1), the Transponder Controller Module (A9), and the Data Simulator (A10). The GPIB Interface Module allows the Macintosh to communicate with each TSPU module by mapping each module to the GPIB bus. The Transponder Controller Module provides the control and status interface to the transponder for bench-top testing. The Data Simulator Module generates pseudo-random data streams that can be used to simulate either forward or return link data. The simulated data is useful for both transponder testing and TURFTS selftesting.

\section{CONCLUSIONS}

The Balloon-Class Transponder and TURFTS enable projects, such as the LDBP, previously unable to afford TDRSS support to gain the benefits of TDRSS usage. Despite the lower cost, the new equipment provides performance and capabilities beyond the equipment currently available. These design improvements should set new standards for future TDRSS User communications systems and support.

\section{REFERENCES}

[1] Stanford Telecom, Santa Clara,

Design Evaluation Report for the Balloon-Class TDRSS User Transponder.

1992.

[2] S.C. Munroe, D.R. Arsenault, K.E. Thompson and A.L. Lattes, "Programmable, Four-Channel, 128 Sample, 40-Ms/s AnalogTernary Correlator,"

in Proceedings of the IEEE 1989 Custom Integrated Circuits Conference, May 1989, pp. 25.1.1-25.1.4.

[3] J.R. Badger

"TDRSS User RF Test Set (TURFTS)—Transportable TDRSS Ground Support Equipment,"

in 1991 GSFC Research \& Technology Report.

[4] D.J. Zillig,

"Low-Cost TDRSS Communications for NASA's Long Duration Balloon Project,"

in 1991 GSFC Research \& Technology Report.

[5] C. Taveras,

"TURFTS Testing Methodology: Subsystem, System, \& User Transponder Testing,"

Professional Intern Program Report, NASA/GSFC, 1992.

[6] J.T. Drake,

"Development of TDRSS Transponder Test Set Controller for NASA's Long Duration Balloon Program,"

Professional Intern Program Report, NASA/GSFC, 1991.

[7] D.J. Israel,

"The Design of the TURFTS DSP Subsystem,"

Professional Intern Program Report, NASA/GSFC, 1991. 